\title{
Laboreal
}

Volume $16 \mathrm{~N}^{\circ} 1 \mid 2020$

Quando o trabalho real é tabu

\section{Cuando el trabajo real es tabú : introducción}

Quando o trabalho real é tabu : introdução

Quand le travail réel est tabou : introduction

When real work is taboo: introduction

\section{Patricio Nusshold, Carole Baudin y Soledad Nion Celio}

\section{(2penEdition}

\section{Journals}

Edición electrónica

URL: http://journals.openedition.org/laboreal/15713

DOI: $10.4000 /$ laboreal. 15713

ISSN: 1646-5237

\section{Editor}

Universidade do Porto

\section{Referencia electrónica}

Patricio Nusshold, Carole Baudin y Soledad Nion Celio, «Cuando el trabajo real es tabú :

introducción », Laboreal [En línea], Volume 16 N¹ 1 2020, Publicado el 01 julio 2020, consultado el 10 julio 2020. URL : http://journals.openedition.org/laboreal/15713

Este documento fue generado automáticamente el 10 julio 2020.

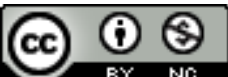

Laboreal está licenciado com uma Licença Creative Commons - Atribuição-NãoComercial 4.0 Internacional. 


\title{
Cuando el trabajo real es tabú : introducción
}

\author{
Quando o trabalho real é tabu : introdução \\ Quand le travail réel est tabou : introduction \\ When real work is taboo: introduction
}

Patricio Nusshold, Carole Baudin y Soledad Nion Celio

1 El trabajo real se revela a partir de los obstáculos. A menudo nos damos cuenta de lo que hacemos a partir del momento en que lo que hacemos fracasa. Redactar la introducción a este número en pleno confinamiento a raíz del Covid-19 agrega a este dossier atípico de la revista Laboreal una dimensión particular. Pascale Molinier decía recientemente en una entrevista que la situación actual puede permitir aprender mucho al personal sanitario (y a nosotros sobre su actividad) del mismo modo que las catástrofes climáticas o las tormentas permiten aprender mucho a los trabajadores de las empresas de gas o de electricidad:

"Se producen en esas circunstancias momentos de intensa movilización de la inteligencia colectiva, los trabajadores escapan del yugo de las normas de calidad, de trazabilidad, de la gestión contable, etc. Van a lo esencial del oficio, e inventan soluciones inéditas - cosas, astucias, combinaciones de todo tipo - para responder ante situaciones desconocidas hasta entonces y que escapan en gran parte a los protocolos. Ante el aumento de los riesgos profesionales del Covid-19, vamos a poder ver a los colectivos unirse y a la vez comunicar permanentemente, cada uno teniendo que cuidar al otro, unos a otros recíprocamente. Paradójicamente, son tiempos de reencuentro con el sentido del oficio, con la importancia de la iniciativa, y de la cooperación. A la vez, tener que estar a la altura de las circunstancias, donde se juega la vida o la muerte, puede llevar a trabajar sin ahorrar energías, con el riesgo evidente a corto o largo plazo de agotamiento físico y colapso moral" (Desriaux, 2020).

2 Podemos decir que hay algo del orden de la confrontación con lo real que permite repensar la actividad.

3 Cuando pensamos hace aproximadamente un año el título de este número, lejos estábamos de imaginar que nos encontraríamos hoy en día confinados en casa, tanto en 
Uruguay, como en Suiza y Francia. Los medios de comunicación hacen referencia a la actividad del personal médico y de enfermería, de lo que hacen para cubrir la brecha entre las prescripciones y las realidades que emergen cotidianamente en su trabajo. Tal vez esta situación tan excepcional visibiliza como nunca antes los fracasos o los intentos exitosos de quienes buscan hacer su trabajo lo mejor posible, sean investigadores o diferentes trabajadores [1] que buscan soluciones en este contexto inédito. Incluso el reconocimiento masivo actual por el trabajo real desarrollado por los equipos sanitarios ante la pandemia, dista mucho de la situación que existía hace solo unos meses.

\section{Trabajo prescripto y trabajo real}

Lo que pareciera haberse hecho relativamente visible son muchas actividades históricamente invisibilizadas, como aquella de los agentes de limpieza, cuidadores, personal sanitario, cajeras de supermercado o educadores. Incluso el trabajo doméstico remunerado que en el período de confinamiento deviene un elemento crítico para organizar el trabajo remoto o desde casa.

5 La ergonomía de la actividad, así como muchas otras disciplinas, tienen como referentes comunes los trabajos de André Ombredane y Jean-Marie Faverge (Ouvrier Bonnaz \& Weill-Fasina, 2015) así como los de Alain Wisner y en particular sus análisis sobre la diferencia entre el trabajo prescrito y el trabajo real (Daniellou, Laville \& Teiger, 1983). La ergonomía de la actividad propone los conceptos de trabajo prescripto, por un lado, como aquello que se pide al trabajador que haga, y por otro lado el de trabajo real como lo efectivo o " actividad» efectiva, aquello que quien trabaja hace realmente (Wisner, 1985, Daniellou, 1996).

Como explica Yves Schwartz (2019) :

“...el concepto de actividad, importante para toda inteligibilidad de nuestra historia e intervención en nuestro presente, es marcado por tres características esenciales : La trasgresión : ninguna disciplina, ningún campo de prácticas puede monopolizar o absorber conceptualmente la actividad; ella atraviesa el consciente y el inconsciente, el verbal y el no verbal, el biológico y el cultural, el mecánico y los valores... La mediación : ella nos impone dialécticas entre todos estos campos, así como entre lo "micro" y lo "macro", lo local y lo global... La contradicción (potencial) : ella es siempre el lugar de debates con resultados siempre inciertos entre las normas antecedentes enraizadas en los medios de vida y las tendencias a la renormalización resingularizadas por los seres humanos."(Op. cit. : pag. 2)

Así, salvar esa distancia entre lo prescripto y lo real es trabajar. La distancia entre lo prescripto y lo real es irreductible y trabajar es llevar adelante en un contexto determinado aquello que se pidió hacer (prescriptivamente). En ese sentido, el abordaje ergonómico se caracteriza por el análisis de la actividad, o sea de todo lo que quienes trabajan hacen en un contexto específico más allá de los procedimientos y las normas que encuadran su tarea. Estos análisis prestan particular atención a la diversidad de la población que trabaja en el marco de los variados dispositivos técnicos y organizacionales. Lejos estamos así de un abordaje tayloriano, y a la noción de "hombre medio" (Wisner, Marcelin \& Briotet, 1971).

8 La ergonomía se focaliza en el análisis de la actividad de cada colectivo de trabajadores en particular buscando comprender las estrategias que se despliegan para cubrir la brecha entre lo prescripto y lo real. $Y$ en ese sentido, hablar del trabajo real nunca es 
fácil : muchas veces quienes trabajamos no somos conscientes de todo lo que hacemos para llevar adelante la tarea. Existe además una dimensión riesgosa al poner en visibilidad la complejidad del trabajo real, las dudas que se ponen en juego, su dimensión incluso subversiva para inventar soluciones que no respetan ciertas normas, para ir más allá de las prescripciones y cumplir con los objetivos o funciones.

Cuando propusimos este dossier para pensar las situaciones en las que el trabajo real es un tabú sabíamos que se trataba de un título que interpelaba. Si volvemos sobre la definición etimológica propuesta por el diccionario de la Real Academia Española (2020) encontramos que la palabra tabú proviene del polinesio y reenvía a la idea de lo prohibido. Se trata de una noción en relación a la prohibición de mencionar, tocar o acercarse a un ser o cosa considerada peligrosa o impura. De cierto modo, la invitación del dossier es cuestionarnos acerca del carácter arriesgado, o incluso a veces asqueroso, obsceno o procaz, que puede constituir en ciertos contextos actuales la referencia a un trabajo real.

\section{Lo real, ese escurridizo objeto de investigación}

Sabemos que los investigadores y consultores que ubican la actividad real en el corazón de sus análisis e intervenciones encuentran a menudo numerosas dificultades para llevar adelante sus proyectos. La brecha entre el trabajo prescripto y el trabajo real no siempre forma parte de las evidencias de sus interlocutores. Durante el análisis de la demanda - punto de partida de todo proceso - y a lo largo de toda la intervención, gran parte del trabajo de los investigadores consiste en dejar en evidencia lo real del trabajo para unos y otros, sea o no este el objetivo central de la investigación o consulta inicial. Pero llevar adelante la empresa de poner en debate lo real del trabajo es complejo, porque lo real se escapa, desliza, se calla, se esconde, o no se puede formular en palabras porque muchas veces es el cuerpo que sabe cómo trabajar más que el lenguaje.

11 Toda actividad humana es completamente imposible de anticipar, y las reglas que intentan normalizar son inevitablemente revisadas y/o redefinidas en el ejercicio de la misma actividad. Describir una actividad supone entonces asumir que las prescripciones no bastan y que las organizaciones funcionan, al fin y al cabo, gracias al trabajo efectivo de los operadores. A raíz de esto, analizar, hablar y debatir sobre el trabajo real no es necesariamente fácil de iniciar o de profundizar, sobre todo en ciertos contextos.

Concomitantemente, este abordaje de la actividad tiene exigencias metodológicas : se trata de arraigar las intervenciones en lo que constituye lo real de las situaciones de trabajo, y el conocimiento de éste exige debatirlo con quienes se trabaja, incluidos quienes tienen poder de decisión. Estos debates parecen evolucionar, a la hora del New Public Management (Chappoz \& Pupion, 2012), donde la gobernanza a través del número prima y pareciera que todo lo que no se puede medir no existe (Supiot, 2015). Los intercambios se vuelven en ciertos contextos ríspidos, complejos o incluso imposibles, sobre todo en algunos sectores como el del cuidado y el trabajo sanitario (Dusmenil, 2011).

13 Sin embargo, muchos investigadores y consultores no bajan los brazos en contextos adversos para pensar el trabajo real a partir de una demanda. Así, el arraigo en la actividad cotidiana de la organización y esta deliberación sobre el trabajo pueden ser concebidos según distintas acepciones y procedimientos particulares : formación al - y 
a través del - análisis del trabajo, auto confrontación cruzada, actividad deóntica, espacios de discusión o de debate sobre el trabajo, grupos de encuentro de trabajo, entre otros. Se trata de variantes que coinciden sin embargo en que el debate contradictorio sobre el trabajo real es indispensable para lograr mejoras en el largo plazo.

Poner en palabras las prácticas reales de las personas genera un problema de prueba. Prueba para quienes trabajan, prueba para quienes deciden hablar, prueba para quienes deciden escuchar, sean colegas, jefes, representantes de personal o consultores. Hablar del trabajo supone un riesgo ligado a develar como trabajamos más allá de las prescripciones, pero además lleva tiempo. Estas cuestiones pueden hacer que quienes nos solicitan, se sientan fácilmente seducidos por modos de producción de conocimientos menos sometidos a los avatares de la vida real y que se presentan como programables en el corto plazo. En particular, muchas organizaciones priorizan los análisis ante los problemas laborales a partir de abordajes exclusivamente cuantitativos sobre los riesgos profesionales, sea bajo la forma de cuestionarios sobre la calidad de vida en el trabajo, encuesta de clima laboral o evaluaciones sobre los riesgos psicosociales (Nusshold, 2015). La riqueza del trabajo real ocupa entonces poco lugar y queda mayoritariamente invisibilizada.

$15 \mathrm{Si}$ pensamos el trabajo real como aquello que es realmente realizado por las trabajadoras y los trabajadores, es necesario reconocer que a menudo ha sido disimulado o incluso escamoteado a lo largo de la historia (Billiard, 2001). De hecho, siempre ha sido difícil para los organizadores del trabajo reconocer la inteligencia práctica de las mujeres y los hombres que hacen funcionar las fábricas y las administraciones (Gernet \& Dejours, 2009). Porque, como decíamos, dar una legitimidad a las habilidades operativas sería reconocer la vulnerabilidad de la prescripción y los fracasos de los sistemas pensados en las oficinas de métodos. Así, como decíamos, la ergonomía es la disciplina que primero develó este oscuro objeto que es el trabajo real. Operó así el retorno de lo que reprimido de toda organización del trabajo y se convirtió en un objeto de estudio rico y complejo.

tas experiencias de campo nos llevan a pens el pudor y las reservas para hablar del trabajo real se pueden haber visto endurecidas y anquilosadas en las empresas e instituciones. Evocar el trabajo real provoca reacciones que ciertos autores del presente dossier consideran epidérmicas, en particular en los directivos, con quienes parece que sería mejor evitar hablar si uno no quiere correr el riesgo de la descalificación y el escándalo. Aquello que Antoine Duarte (2017) llamó el proceso de "imposibilización" de la deliberación sobre el trabajo real y que representa, según él, la piedra angular de la dominación neoliberal a través del trabajo.

\section{Trabajo y tabú : Violencia, sexualidad y dominación}

17 Este número se encuentra orientado a cuestiones relativas a la acción y la transformación. Nuestro objetivo es retomar, apoyar y brindar una reflexión colectiva acerca de los problemas que dificultan -y a veces impiden- el avance de los proyectos para los cuales el trabajo real no puede ser un tema tabú. Nos hemos preguntado desde la construcción de nuestra propuesta si, finalmente, incluso para los investigadores que analizan el trabajo real no es un tabú hablar de sus fracasos o dificultades para llevar adelante sus acciones de terreno en el contexto actual. Como si hablar de los terrenos 
donde todo se ha desarrollado exitosamente fuese más fácil y menos expuesto. Los textos de colegas franceses, canadienses y uruguayos nos permitirán en este dossier profundizar algunos de estos debates que vienen teniendo lugar en múltiples encuentros académicos desde hace varios años.

Hemos privilegiado textos construidos a partir de experiencias de campo que abordan las dificultades que pudieron convertirse en nuevos puntos de partida - o no - para debatir sobre el trabajo con empleadores, representantes del personal $u$ otros protagonistas.

Dos textos hablan de temas difíciles de abordar por los mismos trabajadores: la violencia y la seducción. Otros dos textos nos proponen pensar las condiciones y dificultades para hablar del trabajo real con la jerarquía.

En primer lugar, el sociólogo Pablo Ezquerra propone un análisis de la actividad en el marco del Instituto Nacional de Inclusión Social Adolescente (INISA) del Uruguay. El objetivo central es analizar un tema que constituye un tabú para muchos trabajadores sociales : el miedo a las agresiones y a la violencia. Los trabajadores que ejecutan medidas para la población en conflicto con la ley penal se ven confrontados a riesgos ligados a situaciones vividas por los adolescentes que fueron condenados por un delito. El autor se interesa en particular por los diferentes significados de la seguridad que coexisten en el trabajo cotidiano y cómo estas diferencias confluyen en el trabajo real.

El segundo texto nos lleva a una actividad bien diferente - el trabajo teatral - y nos propone pensar otro tabú : la seducción. Marie Potiron profundiza en este artículo uno de los puntos centrales de su tesis doctoral que gira en torno a la relación entre trabajo y sexualidad. A partir del material clínico de una investigación en psicodinámica del trabajo con un colectivo de actores franceses, se busca pensar la dimensión de la seducción entre quien actúa y el público, pero también con el director que no solo evalúa el resultado del trabajo, sino que también decide la continuidad o no del actor en cada proyecto en un contexto de precariedad. Develar aquello que los trabajadores hacen para llevar adelante su actividad ¿puede "matar la magia" ?

Los últimos dos textos nos hablan principalmente de las ventajas y dificultades para poner en discusión el trabajo real en actividades ligadas al cuidado o al care (Molinier, 2013), ante direcciones que se interesan principalmente en indicadores cuantitativos. François Aubry e Isabelle Feillou presentan los resultados de estudios llevados adelante entre 2014 y 2017 en Quebec con trabajadores de geriatría. La aceleración del ritmo, el aumento de la carga de trabajo y la distancia del management que impide las discusiones colectivas sobre la calidad son los tres elementos principales que surgen del estudio.

Por último, el texto de Antoine Duarte y Roxane Dejours nos lleva al corazón de una intervención desarrollada con trabajadores de la unidad psiquiátrica de un gran centro hospitalario en Francia. A partir de las peripecias de esta investigación, los autores proponen dejar en evidencia los obstáculos para hablar el idioma de la actividad ante una dirección que dirige a través de indicadores numéricos.

Buscamos interrogar a través del presente dossier las relaciones entre subjetividad y organización del trabajo, para pensar si existe un endurecimiento en la posibilidad de poner en debate el trabajo real. Es decir, si existe un tabú - o tabúes- propios a las diferentes actividades. ¿Podemos decir que es tabú hablar del miedo ante la violencia en el contexto del trabajo social o de las estrategias para seducir en el trabajo teatral ? Y más allá de 
los temas que no podemos hablar entre colegas, ¿existen temas que no deben o no pueden ser debatidos con quienes ocupan otro lugar, sean usuarios o superiores jerárquicos? ¿Podemos pensar que en ciertas organizaciones la discusión sobre lo que efectivamente se hace se ha vuelto imposible? ¿Podemos pensar que hay cosas que deben quedar en la sombra, para que el trabajo real siga siendo eficaz? ¿O que quizás, simplemente no existen las palabras que traduzcan los espesores del trabajo real?

La crisis que atravesamos a raíz del COVID-19 nos prueba cómo lo real siempre termina por irrumpir y poner en dificultad nuestro dominio de una situación de trabajo. Las prescripciones nunca bastan. Si los equipos, los directivos o quienes intervienen no parecen siempre estar dispuestos a hablar del trabajo real, las situaciones que se presentan terminan confrontándonos de algún modo $u$ otro con eso que se intentó dejar en la sombra, como un tabú. Lo real termina por irrumpir, y de modo más dramático allí donde menos pudo ser debatido.

Los textos que encontrarán a continuación buscan poner en relieve la relación entre las transformaciones de la organización del trabajo, en particular desde el giro centrado en la gestión y las vías para poner en debate el trabajo real. Las diferentes posturas de los autores proponen abrir el debate sobre el trabajo real, un debate intelectual, pero también político y ético. Para que las trabajadoras y los trabajadores puedan ocupar un rol activo en la elaboración y la transformación de la organización del trabajo es necesario pensar - y repensar - las condiciones para que los debates abran las vías de una genuina transformación del trabajo al servicio de la emancipación.

Esperamos que disfruten este número y que nuevos caminos se abran para pensar nuestro trabajo real a partir de los obstáculos con los que nos confrontamos en nuestras investigaciones.

[1] El uso de un lenguaje que no discrimine ni marque diferencias entre hombres y mujeres es una preocupación de quienes suscriben. No obstante, no hay acuerdo entre lingüistas sobre la manera de cómo hacerlo en nuestro idioma. En tal sentido y con el fin de evitar la sobrecarga gráfica que supondría utilizar en español o/a para marcar la existencia de ambos sexos, hemos optado -en términos generales- emplear el masculino genérico clásico, en el entendido de que todas las menciones representan tanto a varones como mujeres.

\section{BIBLIOGRAFÍA}

Billiard, I. (2001). Santé mentale et travail : L'émergence de la psychopathologie du travail. París : La Dispute. https://doi.org/10.3917/trav.005.0175

Chappoz, Y. \& Pupion, P. (2012). Le New Public Management. Gestion et management public, volume 1/2(2), 1-3. https://doi.org/10.3917/gmp.002.0001

Daniellou, F. (1996). L'ergonomie en quête de ses principes. Toulouse : Octarès Editions.

Daniellou, F., Laville, A., \& Teiger, C. (1983). Fiction et réalité du travail ouvrier. Paris : Cahiers

Français, 209, 39-45 
Desriaux, F. (2020) “ Avant d'être psychologique, le désarroi des soignants est éthique » Entrevista con Pascale Molinier, professeure de psychologie sociale en la Université Sorbonne Paris Nord. Santé et Travail. París.

Duarte, A. (2017). Défenses et résistance en psychodynamique du travail. Tesis de doctorado en Psicología. Universidad Paris Descartes.

Dumesnil, J. (2011). Art médical et normalisation du soin. Paris cedex 14, France : Presses Universitaires de France. https://doi.org/10.3917/puf.dumes.2011.01

Gernet, I. \& Dejours, C. (2009). Évaluation du travail et reconnaissance. Nouvelle revue de psychosociologie, 8(2), 27-36. https://doi.org/10.3917/nrp.008.0027

Molinier, P. (2013). Le travail du care. Paris : La Dispute, coll. Le genre du monde.

Nusshold, P. (2015). Organisation du travail et souffrance psychique dans les activités de service : le cas des centres d'appel en Argentine. Tesis de doctorado en Psicología. Universidad de Buenos Aires y Conservatoire national des arts et métiers - CNAM, 2015. Français. 〈NNT : 2015CNAM1023).

Ouvrier-Bonnaz, R. \& Weill-Fassina, A. (2015). L'analyse du travail. Ruptures et évolutions. Toulouse : Octarès.

Real Academia Española (2020). Diccionario de la lengua española. Consultado en internet el 4 de abril de 2020. En https://dle.rae.es/tabú

Schwartz, Y. (2005). Actividad. Laboreal [En línea], Volume 1 №1 | 2005, Publicado el 01 diciembre 2005, consultado el 18 octubre 2019. URL : http://journals.openedition.org/laboreal/14277

Supiot, A. (2015). La gouvernance par les nombres. Paris : Fayard.

Wisner, A. (1985). Quand voyagent les usines. París : Syros

Wisner A., Marcelin J., Briotet A., (1971). À quel homme le travail doit-il être adapté ?. Paris, Collection du Laboratoire de physiologie du travail-ergonomie du Conservatoire national des arts et métiers, Rapport n 22 (rééd. en 1976), [ré-édition in Wisner, A. (1995). Réflexions sur l'Ergonomie (pp. 47-56). Toulouse : Octarès Éditions.]

\section{AUTORES}

\section{PATRICIO NUSSHOLD}

Université Paul Valéry Montpellier , 3, Route de Mende, 34199 Montpellier Cedex 5, França patricio.nusshold@univ-montp3.fr

\section{CAROLE BAUDIN}

Haute Ecole Arc Direction, Espace de l'Europe 11, 2000 Neuchâtel, Suíça carole.baudin@he-arc.ch

\section{SOLEDAD NION CELIO}

DS- Facultad de Ciencias Sociales, Universidad de la República, Constituyente 1502, Montevideo, Uruguai soledad.nion@cienciassociales.edu.uy 